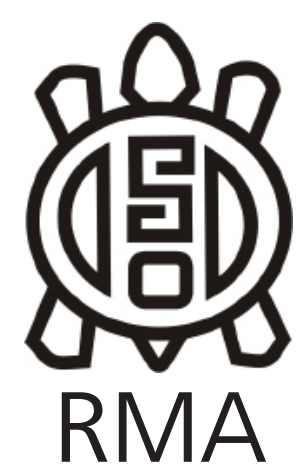

Reseña

Arqueología

\section{Arqueología ambiental: definiciones, prácticas y direcciones ¿encontradas?}

\author{
Environmental Archaeology. Current Theoretical and Methodological \\ Approaches (2018) Evangelia Pişkin, Arkadiusz Marciniak y Marta \\ Bartkowiak (editores). Interdisciplinary Contributions to Archaeology \\ Series. Springer. ISNB 978-3-319-75082-8
}

Este escrito es una reseña del libro "Environmental Archaeology. Current Theoretical and Methodological Approaches" editado por Evangelia Pişkin, Arkadiusz Marciniak y Marta Bartkowiak como parte de la serie Interdisciplinary Contributions to Archaeology (editada por Jelmer Eerkens) de la editorial Springer. El libro tuvo su origen en una sesión titulada "Environmental archaeology and archaeology: divided we stand (still?)" organizada por Pişkin y Bartkowiak en el marco de la 20va reunión de la European Association of Archaeologists realizada en Estambul (Turquía) en septiembre de 2014.

El objetivo de esta reseña comprende no solo resumir y comentar algunas de sus contribuciones, sino también discutir algunos aspectos epistemológicos, metodológicos y prácticos de esta "subdisciplina" de la arqueología que se ha dado en Ilamar "arqueología ambiental". La importancia de este ejercicio radica en la potencial relevancia de este enfoque en la actualidad como semillero de nuevas ideas en torno a la discusión del Antropoceno y el cambio climático desde la arqueología. Al mismo tiempo, cabe mencionar que en 2020 se conmemoran algunos hitos significativos ligados al surgimiento y empleo del concepto de arqueología ambiental. En primera instancia, se cumplen 30 años de la primera discusión académica sobre el empleo de este término, la cual fuera publicada por Circaea (revista editada por la Association of Environmental Archaeology, con base en el Reino Unido) y que consta de diversos aportes que suceden al artículo de Boyd (1990) en forma de comentarios sobre el mismo. Por otro lado, también se cumplen 20 años de la publicación del libro Environmental Archaeology: Principles and Practice de Dena Dincauze
(2000), el cual constituye una lectura obligada no solo para quienes se sienten interpelados por el concepto de arqueología ambiental, sino también para quienes trabajan con reconstrucciones paleoambientales o se interesan en el estudio de los procesos de formación de sitio.

Justamente, considerando la "antigüedad" de este concepto, uno de los objetivos del libro editado por Pişkin, Marciniak y Bartkowiak que me dispongo a reseñar aquí es el de renovar la discusión sobre el uso del término arqueología ambiental a partir de los muchos años desde su aplicación y empleo como disciplina subsidiaria a la arqueología "mainstream", entendida aquí como "convencional". Sin embargo, si bien los editores del libro han realizado un buen trabajo reuniendo escritos sobre distintos temas y cubriendo diversas problemáticas arqueológicas a nivel global, considero que este objetivo resulta algo ambicioso de cara al resultado obtenido, aunque bien vale la pena resaltar y discutir alguno de los puntos desarrollados dentro de sus 248 páginas.

En términos formales, el libro consta de 11 capítulos organizados de manera temática y se encuentra fundamentalmente enfocado en el desarrollo de ciertas metodologías que se engloban bajo el concepto de arqueología ambiental. Es así como, además de incluir algunos capítulos de corte más epistemológico relacionados con los alcances y objetivos de esta subdisciplina, el libro también comprende descripciones de la historia y los avances de campos de estudio como la arqueobotánica, la arqueogenética y la antracología. Finalmente, los últimos capítulos del volumen discuten algunos casos de estudio que abarcan problemáticas tales 
como el pastoreo desde una perspectiva etnoarqueológica, la explotación de recursos ribereños en un área inundable y el uso del espacio intra-sitio en áreas del globo tan disímiles como Chipre, China y Turquía.

Como puede observarse a partir de lo vertido en el párrafo anterior, el grueso del libro se enfoca en cuestiones metodológicas y casos de estudio, dedicando poco espacio - solo los dos primeros capítulos - a la discusión epistemológica de los principios que definen a la arqueología ambiental como una disciplina separada de la arqueología convencional. En el primer capítulo, Evangelia Pişkin y Marta Bartkowiak recopilan diversos antecedentes del surgimiento de la arqueología ambiental como campo de estudio dentro de la arqueología. En esta compilación se observa que el surgimiento de la misma viene ligado a la necesidad de contextualizar los hallazgos arqueológicos, dejando atrás una concepción más bien "anticuarista" de la disciplina. Si bien las autoras exponen diversas definiciones para la arqueología ambiental tomadas de otros/as autores/as, se niegan a darle una definición propia, empleando una metáfora poco convincente: "nobody knows why a rose is called a rose but everybody knows what a rose is" (p. 10). De alguna manera, la existencia de profusa literatura relacionada con el campo de estudio y los alcances de esta "subdisciplina" parece argumentar en contra de esta postura, reclamando la adopción de una teoría (y por ende una definición) o remarcando la necesidad de abandonar este concepto por completo (véase por ejemplo Thomas 2001).

Otro de los puntos polémicos del capítulo redactado por Pişkin y Bartkowiak es el de los distintos tipos de "alienación" que se encuentran en el campo de la arqueología ambiental según las autoras, a saber: 1) alienación geográfica: hace referencia a la existencia de países "que llevan la delantera" donde la arqueología ambiental como disciplina científica se encuentra muy desarrollada en oposición a otros países "menos desarrollados" donde la arqueología ambiental rara vez es incluida en los proyectos; 2) alienación periódica: quienes trabajan con materiales de mayor profundidad temporal suelen estar más involucrados en la arqueología ambiental que aquellos que trabajan en periodos más recientes; 3) alienación interna: hace referencia a la falta de integración entre las distintas "ramas" de la arqueología ambiental (p. ej., geoarqueología y zooarqueología); 4) alienación en la investigación: señala la oposición entre la arqueología desarrollada por universidades u organismos gubernamentales y la arqueología de contrato, en el sentido de que la primera se plantea objetivos y problemas de investigación de antemano mientras que la segunda responde a las necesidades de un privado y comprende principalmente tareas de "salvataje"; 5) alienación de la sociedad: a pesar de la responsabilidad ética de no permanecer indiferentes y a-políticos. Desde nuestra posición como arqueólogos/as sudamericanos/ as podríamos argumentar en contra del primer tipo de "alienación", en la medida en la que la llamada arqueología ambiental se encuentra bastante desarrollada en países "en desarrollo" como Argentina o Chile, donde desde hace décadas que se vienen realizando estudios zooarqueológicos o reconstrucciones paleoambientales de validez internacional. Sin embargo, aquí me interesa destacar el punto menos desarrollado dentro del listado expuesto por Pişkin y Bartkowiak, que es la falta de vinculación con la "sociedad" entendida no solo como el público general susceptible de estar interesado en conocer los detalles de hallazgos sorprendentes, sino también la falta de involucramiento en políticas de gestión del patrimonio. Por supuesto que existen iniciativas que apuntan a remediar este tipo de "alienación", destinadas a incrementar los lazos con el público y con los encargados de las políticas públicas de cuidado, preservación y puesta en valor de los hallazgos arqueológicos. Sin embargo, esta no parecería ser una preocupación de nuestra disciplina en general, algo que se destaca aún más a partir de la falta de desarrollo de este punto en el propio libro reseñado aquí.

El concepto de "alienación" referido por Pişkin y Bartkowiak y su aplicación al campo de la arqueología ambiental tiene una pequeña mención en un trabajo previo de Albarella (2001) publicado en un libro sobre el tema en cuestión que fuera editado por él mismo. Este investigador además es el autor del segundo capítulo del libro reseñado aquí, el cual se destaca por la riqueza de su discusión epistemológica. En dicho capítulo, Albarella retoma una serie de observaciones muy pertinentes destacadas por varios de los colaboradores del libro que editó en 2001 (véase por ejemplo Albarella 2001 y Thomas 2001). Este autor considera que la existencia de ramas de la arqueología como la zooarqueología o la arqueobotánica resulta de utilidad en la medida que ellas se definen en función de los materiales que estudian, cuyos restos requieren conocimientos específicos para ser analizados. Sin embargo, no puede decirse lo mismo de la arqueología ambiental, dado que esta no cuenta con un objeto de estudio propio según este autor. Por esto mismo, Albarella considera que no existe como campo de estudio separado de la "arqueología convencional", estableciendo que el estudio de la interacción entre cultura/sociedad y naturaleza/ambiente es un tema fundamental a toda la disciplina arqueológica, sea que quien se interesa por ello estudie huesos de animales o tiestos cerámicos. Hacia el final del capítulo el autor, entonces, desestima el empleo del término "arqueología ambiental", entendiéndolo como un producto de una mala interpretación de los objetivos de la arqueología y del lugar del ser humano en el mundo. En este sentido, según Albarella (2001) contemplar a la arqueología ambiental como una rama separada de la arqueología convencional parece una mera reposición deslucida de la vieja dicotomía naturaleza-cultura, donde se entiende al "ambiente" como algo separado de la experiencia 
humana, obviando el hecho de que en mayor o menor medida todos los ambientes, pasados y actuales, son constructos culturales.

Volviendo al conjunto de los escritos que integran el volumen "Environmental Archaeology. Current Theoretical and Methodological Approaches" vale la pena resaltar el aporte de algunos de los capítulos más específicos en términos de lecturas actualizadas sobre diferentes "ramas" de la arqueología. Dentro de estos capítulos, resulta de interés aquel que versa sobre arqueogenética, el cual presenta una interesante síntesis sobre la historia de los desarrollos más importantes dentro de esta subdisciplina. En este capítulo Ophélie Lebrasseur, Hannah Ryan y Cinthia Abbona se explayan sobre los preconceptos y las confusiones más comunes en este campo de estudio para luego discutir las consideraciones prácticas necesarias antes de llevar a cabo este tipo de análisis. Finalmente, las autoras recomiendan la existencia de un diálogo constante entre arqueólogos/as y genetistas a fin de llevar estos análisis a buen puerto y evitar la destrucción innecesaria de material de alto valor patrimonial. Por otro lado, también se destaca el capítulo dedicado a la antracología, en donde Ceren Kabukcu hace hincapié en el valor que tiene el material vegetal carbonizado recuperado en los sitios arqueológicos al estudiar los efectos de la acción humana en el paisaje. Luego de pasar revista a la historia de esta subdisciplina, la autora se explaya sobre diversos ejemplos de casos etnográficos donde se obtienen datos de recolección de leña en diversas sociedades según diferentes criterios. Estos casos le permiten discutir en profundidad las diferencias en el manejo que estas sociedades hicieron de los recursos leñosos en pie en el pasado. Otro de los interesantes aportes de este volumen es el que se desarrolla en el cuarto capítulo, donde Kurt J. Gron y Peter Rowley-Conwy se explayan sobre los antecedentes escandinavos de la llamada arqueología ambiental. En este capítulo, los autores exponen los primeros trabajos sobre procesos de formación de sitios desarrollados en diversos sectores de Escandinavia (principalmente Dinamarca), los cuales representaron un hito en la construcción de cronologías precisas para las sucesivas glaciaciones sucedidas en Europa hacia fines del Pleistoceno. Al mismo tiempo, este capítulo toca diversos temas y problemáticas, tales como la ocupación humana en ambientes inundables y la expansión de la agricultura, desde diferentes perspectivas metodológicas como el estudio de varvas o los análisis polínicos.

Luego de estas exposiciones de características tan diversas, resulta extraño que los editores del libro no hayan incluido un capítulo de cierre que se disponga a integrar todos los temas y casos de estudio analizados en los capítulos precedentes, retomando nuevamente la discusión del concepto de arqueología ambiental bajo una nueva óptica. En este sentido, llama la atención la ausencia de un capítulo que presente y discuta nuevas direcciones en las metodologías mencionadas a lo largo del volumen y su relación con problemáticas de relevancia actual, como el cambio climático. A mí entender hubiera sido interesante ampliar la discusión sobre el concepto de arqueología ambiental no solo en términos de sus alcances y limitaciones, sino también en relación al término "humanidades ambientales" (environmental humanities en inglés), acuñado recientemente para hacer referencia a un campo de estudio interdisciplinario donde se reúnen las ciencias sociales y naturales a fin de enfrentar la crisis ecológica actual desde una perspectiva novedosa que integra aspectos éticos, filosóficos, políticos, sociales y biológicos (Emmett y Nye 2017).

Para finalizar, solo resta preguntarse en qué medida resulta útil continuar empleando el concepto de arqueología ambiental. En función de lo relevado aquí y retomando lo planteado por otros autores (véase Albarella 2001 y Thomas 2001) la respuesta pareciera ser un no rotundo. En primera instancia, no existe un consenso claro sobre qué es la arqueología ambiental, sino más que un compendio de metodologías prestadas de otras disciplinas científicas que bien podrían agruparse en torno a conceptos más específicos como zooarqueología o arqueobotánica. Entonces, ¿acaso el empleo de este concepto permite integrar de manera más acabada los resultados alcanzados por quienes trabajan en estos campos de estudio más específicos? Retomando la "alienación" planteada por las propias Pişkin y Bartkowiak en el primer capítulo del volumen la respuesta vuelve a ser negativa. De todas maneras, podemos preguntarnos entonces, ¿resulta útil hablar de arqueología ambiental en el contexto del cambio climático actual, donde se impone la necesidad de actuar rápidamente para frenar sus efectos sobre el planeta? En la medida en que el concepto de arqueología ambiental solo parece generar más discusiones teóricas que acciones concretas, probablemente no. Es por ello por lo que personalmente coincido con el planteo de Thomas (2001): "environmental archaeology is dead". Ahora solo nos resta ahora tomar cartas en el asunto y otorgar profundidad temporal a la discusión global sobre el cambio climático, probablemente el problema más apremiante de todos los mencionados aquí.

\section{Bibliografía citada}

Albarella, U. (2001). Exploring the real nature of environmental archaeology. In U. Albarella (Ed.), Environmental archaeology: Meaning and purpose (pp. 3-13). Dordrecht: Kluwer Academic Publishers.

Boyd, W. E. (1990). Towards a conceptual framework for environmental archaeology: environmental archaeology as a key to past geographies, Circaea 7(2),63-68. 
Dincauze, D. F. (2000). Environmental archaeology: Principles and practice. Cambridge: Cambridge University Press.

Emmett, R. S., and D. E. Nye. (2017). The Environmental Humanities: A Critical Introduction. Cambridge: MIT Press.
Thomas, K. (2001). Environmental archaeology is dead: Long live bioarchaeology, geoarchaeology and human palaeoecology. A comment on "environmental archaeology is not human palaeoecology". In U. Albarella (Ed.), Environmental archaeology: Meaning and purpose (pp. 55-58). Dordrecht: Kluwer Academic Publishers. 